\title{
Fast Molecular-Dynamics Simulation for Ferroelectric Thin-Film Capacitors Using a First-Principles Effective Hamiltonian
}

\author{
Takeshi Nishimatsu ${ }^{1,2}$, Umesh V. Waghmare ${ }^{3}$, Yoshiyuki Kawazoe ${ }^{2}$, and David Vanderbilt ${ }^{1}$ \\ ${ }^{1}$ Department of Physics and Astronomy, Rutgers University, \\ 136 Frelinghuysen Road, Piscataway, NJ 08544-8019 \\ ${ }^{2}$ Institute for Materials Research (IMR), Tohoku University, Sendai 980-8577, Japan \\ ${ }^{3}$ Theoretical Sciences Unit, Jawaharlal Nehru Centre for Advanced \\ Scientific Research (JNCASR), Jakkur, Bangalore, 560 064, India
}

\begin{abstract}
A newly developed fast molecular-dynamics method is applied to $\mathrm{BaTiO}_{3}$ ferroelectric thin-film capacitors with short-circuited electrodes or under applied voltage. The molecular-dynamics simulations based on a first-principles effective Hamiltonian clarify that dead layers (or passive layers) between ferroelectrics and electrodes markedly affect the properties of capacitors, and predict that the system is unable to hop between a uniformly polarized ferroelectric structure and a striped ferroelectric domain structure at low temperatures. Simulations of hysteresis loops of thin-film capacitors are also performed, and their dependence on film thickness, epitaxial constraints, and electrodes are discussed.
\end{abstract}

PACS numbers: 77.80.Dj, 77.80.Fm, 64.70.Nd

\section{INTRODUCTION}

Ferroelectric thin films are beginning to see wideranging applications, for example in multilayer capacitors, nonvolatile FeRAMs, $\stackrel{1}{=}$ and nanoactuators. There is strong pressure to reduce the sizes of such thin-film structures. In recent years, the preparation of oxide thin films by low-temperature non-equilibrium techniques such as molecular beam epitaxy and pulsed-laser deposition have attracted a great deal of attention, as they enable finely controlled growth of epitaxial thin films. ${ }^{2}$

It is well known that the properties of ferroelectric capacitors are highly influenced by the properties of the interface between the ferroelectrics and the electrodes. For example, the fatigue of ferroelectric capacitors is associated with the appearance of dead layers (or passive layers) near the electrodes,$\frac{3,4,5,6}{,}$ and imperfect electrodes cannot fully screen the polarization of ferroelectrics $\underline{7.8}$ leading to a finite depolarization field in the ferroelectric film. However, the nanosize effects and temperature dependences of ferroelectric capacitor hysteresis, polarization switching, and dynamics of domain wall motion remain poorly known. Experimentally, in situ observations are difficult. Theoretically, the long-range Coulomb interaction limits the size and time of molecular-dynamics (MD) simulations, and it has been unclear how to include surface effects and depolarization fields caused by interface structures.

In 1994, King-Smith and Vanderbilt studied the totalenergy surface for zone-center distortions of perovskitetype ferroelectric oxides $A B \mathrm{O}_{3}$ ( $A$ is a monovalent or divalent cation and $B$ is a penta- or tetravalent metal) at zero temperature using first-principles calculations with ultrasoft-pseudopotentials and a plane-wave basis set. $\underline{\underline{9}}$ Starting from the full symmetric cubic perovskite structure, they define the displacements $v_{\alpha}^{\tau}$ of atoms $\tau(=A$, $\left.B, \mathrm{O}_{\mathrm{I}}, \mathrm{O}_{\mathrm{II}}, \mathrm{O}_{\mathrm{III}}\right)$ in the Cartesian directions $\alpha(=x, y, z)$ along the $\Gamma_{15}$ soft-mode normalized direction vectors $\boldsymbol{\xi}_{\alpha}$ as

$$
\boldsymbol{v}_{\alpha}=\left(\begin{array}{c}
v_{\alpha}^{A} \\
v_{\alpha}^{B} \\
v_{\alpha}^{O_{I}} \\
v_{\alpha}^{O_{I I}} \\
v_{\alpha}^{O_{I I I}}
\end{array}\right)=u_{\alpha} \boldsymbol{\xi}_{\alpha}=u_{\alpha}\left(\begin{array}{c}
\xi_{\alpha}^{A} \\
\xi_{\alpha}^{B} \\
\xi_{\alpha}^{O_{I}} \\
\xi_{\alpha}^{O_{I I}} \\
\xi_{\alpha}^{O_{I I I}}
\end{array}\right)
$$

with the scalar soft-mode amplitude $u_{\alpha}$. Under the condition that the strain components $\eta_{i}(i=1, \ldots, 6$; Voigt notation; $\left.\eta_{1}=e_{11}, \eta_{4}=e_{23}\right)$ minimize the total energy for each $\boldsymbol{u}=\left(u_{x}, u_{y}, u_{z}\right)$, they expressed the total energy as

$$
E^{\text {tot }}=E^{0}+\kappa u^{2}+\alpha^{\prime} u^{4}+\gamma^{\prime}\left(u_{x}^{2} u_{y}^{2}+u_{y}^{2} u_{z}^{2}+u_{z}^{2} u_{x}^{2}\right),
$$

where $u^{2}=u_{x}^{2}+u_{y}^{2}+u_{z}^{2}, E^{0}$ is the total energy for the cubic structure, $\kappa$ is half the eigenvalue of the soft mode, and $\alpha^{\prime}$ and $\gamma^{\prime}$ are the constants determined from coupling constants between atomic displacements and strains. Their expression properly describes the coupling of polar atomic-displacement and strain degrees of freedom.

In 1994-1997, Zhong, Vanderbilt, and Rabe ${ }^{10,11}$ and Waghmare and Rabe $\frac{12}{2}$ expanded Eq. (2) from a meanfield framework to a local-mode framework, replacing $\boldsymbol{u}$ by $\{\boldsymbol{u}\}$, where the braces \{\} denote a set of $\boldsymbol{u}$ in a simulation supercell, as

$$
\begin{aligned}
E^{\text {tot }}= & V^{\text {self }}(\{\boldsymbol{u}\})+V^{\text {dpl }}(\{\boldsymbol{u}\})+V^{\text {short }}(\{\boldsymbol{u}\}) \\
& +V^{\text {elas }}\left(\eta_{1}, \cdots, \eta_{6}\right)+V^{\text {int }}\left(\{\boldsymbol{u}\}, \eta_{1}, \cdots, \eta_{6}\right) .
\end{aligned}
$$

Here $V^{\text {self }}, V^{\text {dpl }}, V^{\text {short }}, V^{\text {elas }}$, and $V^{\text {int }}$ are a localmode self-energy, a long-range dipole-dipole interaction, a short-range interaction between soft-modes, an elastic energy, and an interaction between the local modes and local strain, respectively. They employed Eq. (3) as an effective Hamiltonian for $\{\boldsymbol{u}\}$ in the supercell, 
performed Monte-Carlo simulations, and demonstrated the ability to describe the phase transitions of bulk ferroelectrics. The coarse-graining that reduces the 15-dimensional atomic displacement vector $v_{\alpha}^{\tau}$ to a 3dimensional local soft-mode amplitude vector $u_{\alpha}$ in each unit cell was shown to be a good approximation. However, the computation of $V^{\text {dpl }}$ was still time-consuming, owing to the long-range Coulomb interaction, thus limiting system size and simulation time that could be handled in practical simulations.

In 2003, Waghmare, Cockayne, and Burton introduced a technique to decrease the computational time for $V^{\text {dpl }}$ (or forces exerted on $\{\boldsymbol{u}\}$ ) $\stackrel{13}{\underline{b}}$ Direct calculation of the forces in real space requires a computational time proportional to $N^{2}$, i.e., $O\left(N^{2}\right)$, where $N$ is the supercell size $\left(N=L_{x} \times L_{y} \times L_{z}\right)$. It decreases to $O(N \log N)$ if one calculates the forces in reciprocal space using fastFourier transform (FFT) methods. This acceleration in computational speed enabled us to perform MD simulations on $\{\boldsymbol{u}\}$ in a large supercell, and was applied to bulk relaxor ferroelectrics 13,14

Here, we explain how the fast MD method for simulating a first-principles effective Hamiltonian can be applied to study ferroelectric thin-film capacitor structures with short-circuited electrodes or external electric fields. This new MD method can simulate perovskite-type ferroelectric thin-film capacitors with dead layers and consequent depolarization fields. The high speed of this MD method enables us to simulate a ferroelectric material for a realistic system size (up to $100 \mathrm{~nm}$ ) and a realistic time span (>1 ns).

In the next section, we explain the formalism of the new MD-simulation method for thin-film capacitors. Results of simulations of $\mathrm{BaTiO}_{3}$ bulk and thin-film capacitors are shown in Sec. III. In subsection ПII, we confirm the reliability of our MD program by simulating thermal properties of bulk $\mathrm{BaTiO}_{3}$. The advantage of this MD method compared to the Monte-Carlo method is also discussed. In subsection IIIB, we perform heating-up and cooling-down simulations for thin-film $\mathrm{BaTiO}_{3}$ capacitors with perfect and imperfect electrodes. Thickness dependence of simulated striped domain structures in thin-film capacitors with imperfect electrodes are analyzed in detail. We have already reported some simulated results of thin-film capacitors of this subsection and determined thermal properties in Ref. 15] briefly. In subsection IIC newly obtained simulated results of hysteresis loops of thin-film capacitors are reported. In Sec. IV] we summarize the paper.

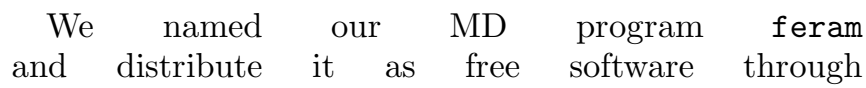
http://loto.sourceforge.net/feram/.

\section{FORMALISM AND METHOD OF CALCULATION}

\section{A. Effective Hamiltonian}

The effective Hamiltonian used in the present MD simulations is basically the same as that in Ref. [13]. Here, we present the Hamiltonian with a notation similar to that in Ref. [11] as

$$
\begin{gathered}
H^{\text {eff }}=\frac{M_{\text {dipole }}^{*}}{2} \sum_{\boldsymbol{R}, \alpha} \dot{u}_{\alpha}^{2}(\boldsymbol{R})+\frac{M_{\text {acoustic }}^{*}}{2} \sum_{\boldsymbol{R}, \alpha} \dot{w}_{\alpha}^{2}(\boldsymbol{R}) \\
+V^{\text {self }}(\{\boldsymbol{u}\})+V^{\mathrm{dpl}}(\{\boldsymbol{u}\})+V^{\text {short }}(\{\boldsymbol{u}\}) \\
+V^{\text {elas, homo }}\left(\eta_{1}, \cdots, \eta_{6}\right)+V^{\text {elas, inho }}(\{\boldsymbol{w}\}) \\
+V^{\text {coup }, \text { homo }}\left(\{\boldsymbol{u}\}, \eta_{1}, \cdots, \eta_{6}\right)+V^{\text {coup }, \text { inho }}(\{\boldsymbol{u}\},\{\boldsymbol{w}\}) \\
-Z^{*} \sum_{\boldsymbol{R}} \mathcal{E} \cdot \boldsymbol{u}(\boldsymbol{R}),
\end{gathered}
$$

where $\boldsymbol{u}=\boldsymbol{u}(\boldsymbol{R})$ and $\boldsymbol{w}=\boldsymbol{w}(\boldsymbol{R})$ are, respectively, the local soft-mode amplitude vector and the local acoustic displacement vector of the unit cell at $\boldsymbol{R}$, the $\alpha$ component of $\boldsymbol{R}$ runs over

$$
R_{\alpha}=0, a_{0}, 2 a_{0}, \cdots\left(L_{\alpha}-1\right) a_{0},
$$

$\eta_{1}, \cdots, \eta_{6}$ are the homogeneous strain components, and $M_{\text {dipole }}^{*}$ and $M_{\text {acoustic }}^{*}$ are the effective masses for $\boldsymbol{u}$ and $\boldsymbol{w}$, respectively. Note that $\boldsymbol{u}$ can also be considered as the optical displacement, in contrast to the acoustic displacement $\boldsymbol{w}$, or the dipole moment $Z^{*} \boldsymbol{u}$, where $Z^{*}$ is the Born effective charge associated with the soft mode. In the effective Hamiltonian (4), external electric field $\mathcal{E}$ is taken into account through its vector product with each dipole moment $Z^{*} \boldsymbol{u}$.

To determine the effective mass $M_{\text {dipole }}^{*}$, let $\epsilon_{\alpha}^{\tau}(\boldsymbol{k}, i)$ be a mass-weighted $i$-th eigenvector of the phonon dynamical matrix at wavevector $\boldsymbol{k}$. Its eigenvalue $\{\omega(\boldsymbol{k}, i)\}^{2}$ is the corresponding phonon frequency. Moreover, let $d_{\alpha}^{\tau}(\boldsymbol{k}, i)=\epsilon_{\alpha}^{\tau}(\boldsymbol{k}, i) / \sqrt{M_{\tau}}$ be an atomic displacement vector, which is normalized as $\sum_{\alpha, \tau}\left\{d_{\alpha}^{\tau}(\boldsymbol{k}, i)\right\}^{2}=1$ by adjusting the norm of $\boldsymbol{\epsilon}(\boldsymbol{k}, i)$. Here, $M^{\tau}$ is the mass of atom $\tau$. Generally, the effective mass of a phonon is $\boldsymbol{k}$ and mode-dependent:

$$
M^{*}(\boldsymbol{k}, i)=\sum_{\alpha, \tau}\left\{d_{\alpha}^{\tau}(\boldsymbol{k}, i)\right\}^{2} M^{\tau}
$$

However, as an approximation, we have to employ a unique effective mass for dipoles in the MD simulation. Thus using the steepest descent $\Gamma_{15}$ soft-mode normalized direction vectors $\boldsymbol{\xi}_{z}=(0.20,0.76,-0.21,-0.21,-0.53)$ and $\boldsymbol{\xi}_{x}=\boldsymbol{\xi}_{y}=0$ from Ref. [11], for $\mathrm{BaTiO}_{3}$, we set $M_{\text {dipole }}^{*}$ as

$$
M_{\text {dipole }}^{*}=\sum_{\tau}\left\{\xi_{z}^{\tau}\right\}^{2} M^{\tau}=39.0 \mathrm{amu}
$$


It should be mentioned that $\xi_{z}^{\tau}$ is not equal to the $d_{\alpha}^{\tau}$ of the $\Gamma_{15}$ soft-mode of phonon, because $M^{A}, M^{B}$, and $M^{\mathrm{O}}$ are not identical.

The local-mode self-energy $V^{\text {self }}(\{\boldsymbol{u}\})$ is

$$
\begin{gathered}
V^{\text {self }}(\{\boldsymbol{u}\})=\sum_{i=1}^{N}\left\{\kappa_{2} u^{2}\left(\boldsymbol{R}_{i}\right)+\alpha u^{4}\left(\boldsymbol{R}_{i}\right)+\right. \\
\left.\gamma\left[u_{y}^{2}\left(\boldsymbol{R}_{i}\right) u_{z}^{2}\left(\boldsymbol{R}_{i}\right)+u_{z}^{2}\left(\boldsymbol{R}_{i}\right) u_{x}^{2}\left(\boldsymbol{R}_{i}\right)+u_{x}^{2}\left(\boldsymbol{R}_{i}\right) u_{y}^{2}\left(\boldsymbol{R}_{i}\right)\right]\right\},
\end{gathered}
$$

where $u^{2}\left(\boldsymbol{R}_{i}\right)=u_{x}^{2}\left(\boldsymbol{R}_{i}\right)+u_{y}^{2}\left(\boldsymbol{R}_{i}\right)+u_{z}^{2}\left(\boldsymbol{R}_{i}\right)$.

The long-range dipole-dipole interaction $V^{\mathrm{dpl}}(\{\boldsymbol{u}\})$ is

$$
V^{\mathrm{dpl}}(\{\boldsymbol{u}\})=\frac{1}{2} \sum_{i=1}^{N} \sum_{\alpha} \sum_{j=1}^{N} \sum_{\beta} u_{\alpha}\left(\boldsymbol{R}_{i}\right) \Phi_{\alpha \beta}\left(\boldsymbol{R}_{i j}\right) u_{\beta}\left(\boldsymbol{R}_{j}\right),
$$

where

$$
\Phi_{\alpha \beta}\left(\boldsymbol{R}_{i j}\right)=\frac{Z^{* 2}}{\epsilon_{\infty}} \sum_{\boldsymbol{n}} \frac{\delta_{\alpha \beta}-3\left(\widehat{\boldsymbol{R}_{i j}+\boldsymbol{n}}\right)_{\alpha}\left(\widehat{\boldsymbol{R}_{i j}+\boldsymbol{n}}\right)_{\beta}}{\left|\boldsymbol{R}_{i j}+\boldsymbol{n}\right|^{3}},
$$

$\epsilon_{\infty}$ is the optical dielectric constant (or refractive index squared), $\delta_{\alpha \beta}$ is the Kronecker delta, a hat indicates a unit vector, $\boldsymbol{n}$ is the supercell lattice vector

$$
n_{\alpha}=\cdots,-2 L_{\alpha} a_{0},-L_{\alpha} a_{0}, 0, L_{\alpha} a_{0}, 2 L_{\alpha} a_{0}, \cdots \quad,
$$

and $a_{0}$ is the equilibrium lattice constant. In Eq. (10), $\sum^{\prime}$ indicates that the summation does not include terms for which $\boldsymbol{R}_{i j}=\boldsymbol{n}=0$.

We take account of short-range interactions between the optical displacements $\boldsymbol{u}(\boldsymbol{R})$ up to third nearest neighbor (3nn) as

$$
V^{\text {short }}(\{\boldsymbol{u}\})=\frac{1}{2} \sum_{i=1}^{N} \sum_{\alpha} \sum_{j}^{3 \mathrm{nn}} \sum_{\beta} u_{\alpha}\left(\boldsymbol{R}_{i}\right) J_{i j, \alpha \beta} u_{\beta}\left(\boldsymbol{R}_{j}\right),
$$

where $J_{i j, \alpha \beta}$ is the short-range interaction matrix, which can be classified into 7 independent interaction parameters $\stackrel{11}{\underline{ }} J_{i j, \alpha \beta}= \pm j_{k}(k=1, \cdots, 7)$.

In practice, $\kappa_{2} u_{i}^{2}$ in Eq. (8), Eq. (9), and Eq. (12), in which $u_{\alpha}$ is quadratic, are gathered and calculated in reciprocal space as

$$
V^{\text {quad }}(\{\boldsymbol{u}\})=\frac{1}{2} \sum_{\boldsymbol{k}} \sum_{\alpha, \beta} \widetilde{u}_{\alpha}^{*}(\boldsymbol{k}) \widetilde{\Phi}_{\alpha \beta}^{\mathrm{quad}}(\boldsymbol{k}) \widetilde{u}_{\beta}(\boldsymbol{k}),
$$

where $\widetilde{u}_{\alpha}(\boldsymbol{k})$ is the Fourier transform

$$
\widetilde{u}_{\alpha}(\boldsymbol{k})=\sum_{\boldsymbol{R}} u_{\alpha}(\boldsymbol{R}) \exp (-i \boldsymbol{k} \cdot \boldsymbol{R}),
$$

of $u_{\alpha}(\boldsymbol{R}), \widetilde{\Phi}_{\alpha \beta}^{\text {quad }}(\boldsymbol{k})$ is similarly the Fourier transform of the quadratic interaction matrix (which is only calculated once at the beginning of simulation),$\frac{13}{\Perp}$ and $\boldsymbol{k}$ is a reciprocal vector in the first Brillouin zone of the unit cell such as

$k_{\alpha}=-\frac{L_{\alpha}-1}{2 L_{\alpha}} \frac{2 \pi}{a_{0}}, \cdots,-\frac{1}{L_{\alpha}} \frac{2 \pi}{a_{0}}, 0, \frac{1}{L_{\alpha}} \frac{2 \pi}{a_{0}}, \cdots, \frac{1}{2} \frac{2 \pi}{a_{0}}$. is

The homogeneous elastic energy $V^{\text {elas, homo }}\left(\eta_{1}, \cdots, \eta_{6}\right)$

$$
\begin{aligned}
V^{\text {elas, homo }}\left(\eta_{1}, \cdots, \eta_{6}\right) & =\frac{N}{2} B_{11}\left(\eta_{1}^{2}+\eta_{2}^{2}+\eta_{3}^{2}\right) \\
& +N B_{12}\left(\eta_{2} \eta_{3}+\eta_{3} \eta_{1}+\eta_{1} \eta_{2}\right) \\
& +\frac{N}{2} B_{44}\left(\eta_{4}^{2}+\eta_{5}^{2}+\eta_{6}^{2}\right),
\end{aligned}
$$

where $B_{11}, B_{12}$, and $B_{44}$ are the elastic constants expressed in energy unit $\left(B_{11}=a_{0}^{3} C_{11}, B_{12}=a_{0}^{3} C_{12}\right.$, and $\left.B_{44}=a_{0}^{3} C_{44}\right)$.

The inhomogeneous elastic energy $V^{\text {elas, inho }}(\{\boldsymbol{w}\})$ is also calculated in reciprocal space as

$$
V^{\text {elas, inho }}(\{\boldsymbol{w}\})=\frac{1}{2} \sum_{\boldsymbol{k}} \sum_{\alpha, \beta} \widetilde{w}_{\alpha}^{*}(\boldsymbol{k}) \widetilde{\Phi}_{\alpha \beta}^{\text {elas, inho }}(\boldsymbol{k}) \widetilde{w}_{\beta}(\boldsymbol{k}) .
$$

For the force constant matrix $\widetilde{\Phi}_{\alpha \beta}^{\text {elas, inho }}(\boldsymbol{k})$, we employed the long-wavelength approximation. For instance, the diagonal part is

$$
\widetilde{\Phi}_{x x}^{\text {elas, inho }}(\boldsymbol{k})=\frac{1}{N}\left[k_{x}^{2} B_{11}+k_{y}^{2} B_{44}+k_{z}^{2} B_{44}\right],
$$

and the off-diagonal part is

$$
\widetilde{\Phi}_{x y}^{\text {elas, inho }}(\boldsymbol{k})=\frac{1}{N}\left[k_{x} k_{y} B_{12}+k_{x} k_{y} B_{44}\right] .
$$

The coupling between $\{\boldsymbol{u}\}$ and homogeneous strain is the same as that given in Ref. [9], i.e.,

$$
V^{\text {coup, homo }}\left(\{\boldsymbol{u}\}, \eta_{1}, \cdots, \eta_{6}\right)=\frac{1}{2} \sum_{\boldsymbol{R}} \sum_{i=1}^{6} \sum_{j=1}^{6} \eta_{i} C_{i j} y_{j}(\boldsymbol{R}) \text {. }
$$

Here, $y_{1}(\boldsymbol{R})=u_{x}^{2}(\boldsymbol{R}), y_{2}(\boldsymbol{R})=u_{y}^{2}(\boldsymbol{R}), y_{3}(\boldsymbol{R})=u_{z}^{2}(\boldsymbol{R})$, $y_{4}(\boldsymbol{R})=u_{y}(\boldsymbol{R}) u_{z}(\boldsymbol{R}), y_{5}(\boldsymbol{R})=u_{z}(\boldsymbol{R}) u_{x}(\boldsymbol{R})$, and $y_{6}(\boldsymbol{R})$ $=u_{x}(\boldsymbol{R}) u_{y}(\boldsymbol{R})$,

$$
\mathbf{C}=\left(\begin{array}{cccccc}
B_{1 x x} & B_{1 y y} & B_{1 y y} & 0 & 0 & 0 \\
B_{1 y y} & B_{1 x x} & B_{1 y y} & 0 & 0 & 0 \\
B_{1 y y} & B_{1 y y} & B_{1 x x} & 0 & 0 & 0 \\
0 & 0 & 0 & 2 B_{4 y z} & 0 & 0 \\
0 & 0 & 0 & 0 & 2 B_{4 y z} & 0 \\
0 & 0 & 0 & 0 & 0 & 2 B_{4 y z}
\end{array}\right)
$$

and $B_{1 x x}, B_{1 y y}$, and $B_{4 y z}$ are the coupling coefficients defined in Ref. [9].

The coupling between $\{\boldsymbol{u}\}$ and inhomogeneous strain is also calculated in reciprocal space as

$$
V^{\text {coup , inho }}(\{\boldsymbol{u}\},\{\boldsymbol{w}\})=\frac{1}{2} \sum_{\boldsymbol{k}} \sum_{\alpha} \sum_{i=1}^{6} \widetilde{w}_{\alpha}(\boldsymbol{k}) \widetilde{B}_{\alpha i}(\boldsymbol{k}) \widetilde{y}_{i}(\boldsymbol{k}),
$$


where $\widetilde{w}_{\alpha}(\boldsymbol{k})$ and $\widetilde{y}_{i}(\boldsymbol{k})$ are the Fourier transforms of $w_{\alpha}(\boldsymbol{R})$ and $y_{i}(\boldsymbol{R})$, respectively. For the $3 \times 6$ coupling matrix $\mathbf{B}(\boldsymbol{k})$, we again employed the long-wavelength approximation

$$
\widetilde{\mathbf{B}}(\boldsymbol{k})=\frac{1}{N}\left(\begin{array}{cccccc}
k_{x} B_{1 x x} & k_{x} B_{1 y y} & k_{x} B_{1 y y} & 0 & 2 k_{z} B_{4 y z} & 2 k_{y} B_{4 y z} \\
k_{y} B_{1 y y} & k_{y} B_{1 x x} & k_{y} B_{1 y y} & 2 k_{z} B_{4 y z} & 0 & 2 k_{x} B_{4 y z} \\
k_{z} B_{1 y y} & k_{z} B_{1 y y} & k_{z} B_{1 x x} & 2 k_{y} B_{4 y z} & 2 k_{x} B_{4 y z} & 0
\end{array}\right)
$$

In the present MD simulations of $\mathrm{BaTiO}_{3}$, the parameters from Refs. [10] and 11], which are determined by first-principles calculations, are employed. As mentioned in Refs. [10] and [11], this parameter set leads to an underestimation of the Curie temperature $T_{\mathrm{C}}$. To correct this underestimation, we follow these references in applying a negative pressure of $p=-5.0 \mathrm{GPa}$ in all simulations.

\section{B. Molecular Dynamics}

MD simulations with the effective Hamiltonian of Eq. (41) are performed in the canonical ensemble using the Nosé-Poincaré thermostat 16 This simplectic thermostat is so efficient that we can set the time step to $\Delta t=2 \mathrm{fs}$. In our present simulations, we thermalize the system for 40,000 time steps, after which we average the properties for 10,000 time steps.

In Fig. 1 we roughly illustrate how to calculate the forces exerted on $u_{\alpha}(\boldsymbol{R})$ with $\widetilde{\Phi}_{\alpha \beta}^{\text {quad }}(\boldsymbol{k})$ in Eq. (13) and how the time evolution is simulated. First, $u_{\alpha}(\boldsymbol{R})$ is FFTed to $\widetilde{u}_{\alpha}(\boldsymbol{k})$, the force $\widetilde{F}_{\alpha}(\boldsymbol{k})=-\sum_{\beta} \widetilde{\Phi}_{\alpha \beta}^{\text {quad }}(\boldsymbol{k}) \widetilde{u}_{\beta}(\boldsymbol{k})$ is calculated in reciprocal space, and then the force in real space is obtained by the inverse FFT of $\widetilde{F}_{\alpha}(\boldsymbol{k})$. In practice, updates of $u_{\alpha}(\boldsymbol{R})$ and $\dot{u}_{\alpha}(\boldsymbol{R})=\frac{\partial}{\partial t} u_{\alpha}(\boldsymbol{R})$ are processed in the manner of the Nosé-Poincaré thermostat.

The homogeneous strain components $\eta_{1}, \cdots, \eta_{6}$ are determined by solving

$$
\begin{aligned}
\frac{\partial}{\partial \eta_{i}}\left[V^{\text {elas, homo }}\left(\eta_{1}, \cdots, \eta_{6}\right)\right. & \\
& \left.+V^{\text {coup, homo }}\left(\{\boldsymbol{u}\}, \eta_{1}, \cdots, \eta_{6}\right)\right]=0
\end{aligned}
$$

at each time step according to $\{\boldsymbol{u}\}$ so that $\eta_{1}, \cdots, \eta_{6}$ minimize $V^{\text {elas, homo }}\left(\eta_{1}, \cdots, \eta_{6}\right)+V^{\text {coup, homo }}\left(\{\boldsymbol{u}\}, \eta_{1}, \cdots, \eta_{6}\right)$. While the local acoustic displacement $w_{\alpha}(\boldsymbol{R})$ could be treated as dynamical variables using the effective mass $M_{\text {acoustic }}^{*}$, we have instead chosen to integrate out these variables in a manner similar to the treatment of the homogeneous strain. That is, $w_{\alpha}(\boldsymbol{R})$ is determined so that $V^{\text {elas, inho }}(\{\boldsymbol{w}\})+V^{\text {coup, inho }}(\{\boldsymbol{u}\},\{\boldsymbol{w}\})$ becomes minimum at each time step according to $u_{\alpha}(\boldsymbol{R})$. Technically, the minimization is performed by solving the linear

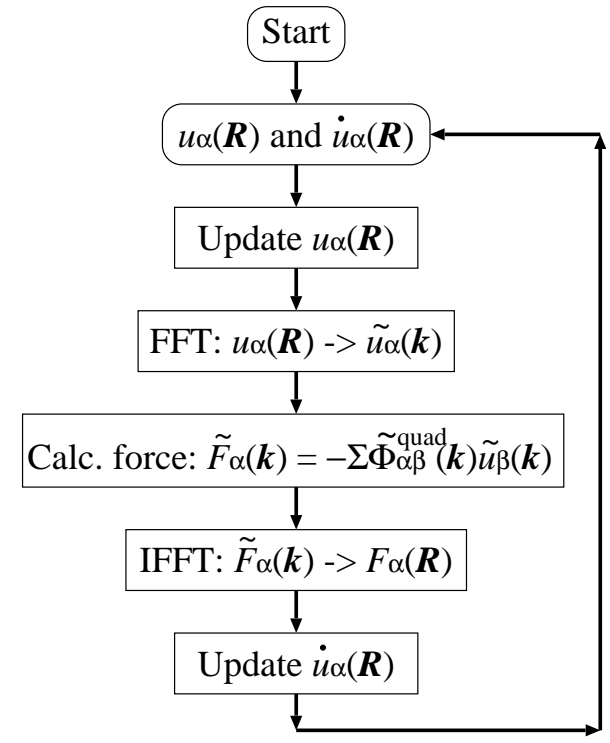

FIG. 1: Simplified flow chart for calculating forces on $u_{\alpha}(\boldsymbol{R})$. Fast Fourier transform (FFT) and inverse FFT (IFFT) enable rapid calculation of long-range dipole-dipole interactions.

set of equations

$$
\widetilde{\Phi}^{\text {elas, inho }}(\boldsymbol{k}) \widetilde{\boldsymbol{w}}(\boldsymbol{k})+\widetilde{\mathbf{B}}(\boldsymbol{k}) \widetilde{\boldsymbol{y}}(\boldsymbol{k})=\mathbf{0}
$$

for each $\boldsymbol{k}$ in reciprocal space.

\section{Ferroelectric Thin Films}

If a ferroelectric thin film is placed in isolation in vacuum without electrodes as depicted in Fig. 2(a), its spontaneous polarization $\boldsymbol{P}=\left(P_{x}, P_{y}, P_{z}\right)$, which is represented by a thick arrow in the figure, induces charges $\pm \sigma_{\text {ind }}= \pm P_{z}$ at both surfaces, and the induced charges cause a full depolarization field in the thin film, $\mathcal{E}_{\mathrm{d}}=$ $-4 \pi \sigma_{\text {ind }} \widehat{z}=-4 \pi P_{z} \widehat{z}$. On the other hand, if the ferroelectric thin film is placed between short-circuited perfect electrodes as depicted in Fig. 2(b), the induced charges are fully canceled by free charges $\sigma_{\text {free }}$ arising at both surfaces of the electrodes, $\mathcal{E}_{\mathrm{d}}=-4 \pi\left(\sigma_{\text {ind }}+\sigma_{\text {free }}\right) \widehat{\boldsymbol{z}}=0$. This geometric circumstance can be simulated with the doubly periodic supercell as depicted in Fig. 2(c), because 
the two electrodes act as two electrostatic mirrors facing each other, and the mirrors make oppositely charged infinite mirror images beyond the electrodes.

We can also introduce dead layers of thickness $d$ between the ferroelectric thin film and electrodes by constraining the local soft-mode amplitudes to vanish $(\boldsymbol{u}=$ 0 ) in these layers, as illustrated in Fig. 2(d). With the dead layers, the infinite mirror images beyond the electrodes become $\frac{l}{l+d}$ more sparse than images of the without-dead-layer configuration. Consequently, the free charges arising at the electrode surfaces decrease to $\sigma_{\text {free }}=-\frac{l}{l+d} \sigma_{\text {ind }}$, where $l$ is the ferroelectric film thickness. This simulates short-circuited imperfect electrodes resulting in a depolarization field of

$$
\mathcal{E}_{\mathrm{d}}=-4 \pi \frac{d}{l+d} P_{z} \widehat{\boldsymbol{z}} .
$$

We can also use a doubly periodic supercell with dead layers for this case. Physically, the depolarization field of Eq. (26) can arise either from the presence of a dead layer in the ferroelectric near the interface, or from imperfect screening at the metal electrode, or both. We can define an effective screening length for each of these effects, and we interpret the "dead-layer thickness" $d$ of our model as corresponding to the sum of these two physical screening lengths. The screening length associated with the electrode interface appears in Eq. (16) of Ref. 7] and Eq. (1) of Ref. [8] and is discussed for the $\mathrm{SrRuO}_{3} / \mathrm{BaTiO}_{3}$ interface in Refs. [17], [18], and 19]. Therefore, while the model does not explicitly incorporate information about the interface screening, this information is effectively included in the definition of the total screening length $d$ in our model. Thus, for example, simulations at constant $d$ for various film thicknesses can give the thickness dependence of the properties of capacitors with a certain interface structure.

In the present MD simulations, the local soft-mode amplitude vectors $\boldsymbol{u}$ in dead layers are fixed to zero by the infinitely large mass. This infinitely-large-mass trick is congenial to the Nosé-Poincaré thermostat for maintaining the Nosé-Poincaré Hamiltonian at zero. Moreover, this treatment also has another advantage in that the short-range interactions between the surfaces of ferroelectric thin film and the electrodes are automatically truncated.

The depolarization field $\mathcal{E}_{\mathrm{d}}$ increases the total energy of the ferroelectric thin film by $-\boldsymbol{P} \cdot \mathcal{E}_{\mathrm{d}}=4 \pi \frac{d}{l+d} P_{z}^{2}$. To avoid forming a depolarization field in ferroelectric thin films, it is known that the films often develop striped domain structures. $20,21,22,23$ The introduction of the striped domain structure can eliminate some part of the energy increase $4 \pi \frac{d}{l+d} P_{z}^{2}$, because $P_{z}$ becomes zero on average. However, the striped domain structure involves an energy cost in the short-range interaction $V^{\text {short }}(\{\boldsymbol{u}\})$, because it has domain boundaries between which $\boldsymbol{u}$ has opposite direction $\pm z$. The shorter the wavelength $\lambda$ of the striped domain structure, the weaker the depolarization field, but the higher the short-range interaction energy. The

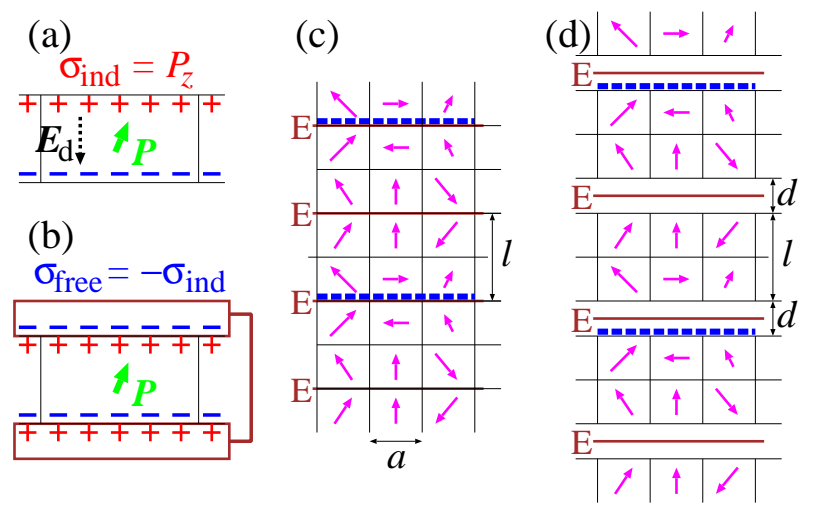

FIG. 2: Schematic illustrations of ferroelectric thin films of thickness $l$ unit cells (here $l=2$ ). (a) Isolated thin film in vacuum. (b) Thin film sandwiched between short-circuited perfect electrodes. Doubly periodic boundary conditions for simulations of films sandwiched between perfect and imperfect short-circuited electrodes are depicted in (c) and (d), respectively. Horizontal thick lines marked with "E" represent the electrostatic mirrors used to model electrodes. They are a distance $d / 2$ away from the ferroelectric film surface $(d=0$ in (c), $d=1$ in (d)). Each thin arrow represents a local dipole within a unit cell $\left(a^{3}=3.94 \AA^{3}\right)$ of the $\mathrm{BaTiO}_{3}$ crystal. Thick dashed lines enclose the periodic cell used for simulations.

ground state of a ferroelectric thin film will be decided by a competition between the long-range dipole-dipole interactions which favor a short-period domain structure, and domain-wall energy that arises from the short-range interactions and favors a uniformly polarized structure or a longer-period striped structure. In some previous works, $24,25,26$ the imperfect screening was mimicked with a parameter. On the other hand, our method with doubly periodic boundary condition does not require any parameters, because the effect of imperfectness of electrodes is automatically and implicitly included in the long-range dipole-dipole interaction $V^{\mathrm{dpl}}(\{\boldsymbol{u}\})$.

\section{RESULTS AND DISCUSSION}

\section{A. Bulk BaTiO}

We first check the reliability of our MD program by comparing results of our simulations for bulk $\mathrm{BaTiO}_{3}$ with earlier work based on the same effective Hamiltonian ${ }^{10,11}$ We used a system size of $L_{x} \times L_{y} \times L_{z}=$ $16 \times 16 \times 16$ and small temperature steps in heatingup $(+5 \mathrm{~K} / \mathrm{step})$ and cooling-down $(-5 \mathrm{~K} / \mathrm{step})$ simulations, with initial configuration generated randomly: $\left\langle u_{\alpha}\right\rangle=0.07 \AA$ and $\left\langle u_{\alpha}^{2}\right\rangle-\left\langle u_{\alpha}\right\rangle^{2}=(0.02 \AA)^{2}$. We have also checked that there was no dependence of results of these simulations on initial configurations. The temperature dependence of the homogeneous strain components (see Fig. 3), which are the secondary order parameters of ferroelectric phase transitions, exhibits the correct se- 


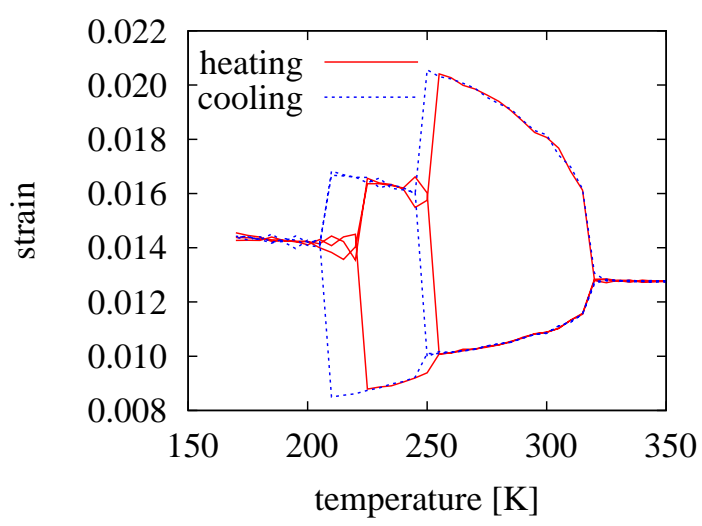

FIG. 3: Average homogeneous strains $e_{x x}, e_{y y}$, and $e_{z z}$ as a function of temperature in heating-up $(+5 \mathrm{~K} /$ simulation, solid lines) and cooling-down ( $-5 \mathrm{~K} /$ simulation, dashed lines) simulations for a $16 \times 16 \times 16$ supercell. Strains are measured relative to the LDA minimum-energy cubic structure with lattice constant $3.948 \AA$.

quence of phase transitions in $\mathrm{BaTiO}_{3}$ known experimentally. Even under the negative pressure $p=-5.0 \mathrm{GPa}$, the paraelectric to ferroelectric transition temperature $T_{\mathrm{C}}$ is underestimated at around $320 \mathrm{~K}$ in comparison with the experimental value of $T_{\mathrm{C}}=408 \mathrm{~K}$. Our estimates of $T_{\mathrm{C}}$ 's agree fairly well with the ones reported in Ref. [11]. The relatively weak first-order nature of the cubic-totetragonal phase transition in comparison with the firstorder tetragonal-to-orthorhombic and orthorhombic-torhombohedral phase transitions is evident in the width of the temperature intervals of hysteresis (see Fig. 30). We note that the ability to simulate time-dependent phenomena is one of advantages of MD simulations compared to Monte-Carlo simulations.

\section{B. $\mathrm{BaTiO}_{3}$ ferroelectric thin-film capacitors}

We now simulate and analyze the behavior of epitaxially grown films of $\mathrm{BaTiO}_{3}$ on $\mathrm{GdScO}_{3}$ substrates $\stackrel{2}{2}$ In our simulations, we represent this with $1 \%$ in-plane biaxial compressive strain by maintaining the homogeneous strain $\eta_{1}=\eta_{2}=-0.01$ and $\eta_{6}=0 . \quad$ In other words, we maintained the average lattice constants $a$ and $b$ at $0.99 a_{0}$ and angle $\gamma$ at $90^{\circ}$. We use supercell sizes of $L_{x} \times L_{y} \times L_{z}=32 \times 32 \times 2(l+d)$ and $40 \times 40 \times 2(l+d)$ and simulate ferroelectric layers of thickness $l$ sandwiched between two short-circuited electrodes with $(d=1)$ and without $(d=0)$ dead layers. This is accomplished through use of doubly periodic boundary conditions as explained earlier.

Both the heating-up and cooling-down simulations are started with an initial configuration of $\left\langle u_{x}\right\rangle=\left\langle u_{y}\right\rangle=0$, $\left\langle u_{z}\right\rangle=0.07 \AA$ and $\left\langle u_{\alpha}^{2}\right\rangle-\left\langle u_{\alpha}\right\rangle^{2}=(0.02 \AA)^{2}$. In the cooling-down simulations, which start at a sufficiently high temperature, the initial configuration changes to an unpolarized one $\left(\left\langle u_{z}\right\rangle=0\right)$ during thermalization. We monitor the temperature dependence of $\left\langle u_{\alpha}\right\rangle$ and $\left\langle u_{\alpha}^{2}\right\rangle$ for thin films with thicknesses $l=15,31,127$, and 255 with dead layers $d=1$ and a thin film with thickness $l=32$ without dead layers $(d=0)$ (see Fig. [ and animations in the EPAPS 27 ). The behavior of the film with no dead layer is the same in heating and cooling simulations. In contrast, for the films with a dead layer $(d=1)$, the transition behavior exhibited by $\left\langle u_{z}\right\rangle=0$ is rather different in heating and cooling simulations, although the temperature dependence of $\left\langle u_{z}^{2}\right\rangle$ is almost the same in both the kinds of simulations. In the heating-up simulations, the discontinuity in $\left\langle u_{z}\right\rangle$ as a function of temperature marks a transition from a ferroelectric state with almost uniform out-of-plane polarization (Fig. 5 (a)) to one with a striped domain structure (Fig. 5(b) and (c)). We find that this transition temperature, $T_{\mathrm{S}}(l, d=1)$, exhibits a strong dependence on size $l$. We note that this transition is missing in the cooling-down simulations; just above $T_{\mathrm{S}}$, striped domain structures appear and the stripes remain and be frozen at $T<T_{\mathrm{S}}$. The temperature $T_{\mathrm{C}}(l, d=1)$ at which $\sqrt{\left\langle u_{z}^{2}\right\rangle}(T)$ exhibits a change in its slope marks another transition, namely from a striped domain phase to a paraelectric phase. $T_{\mathrm{C}}(l, d=1)$ depends relatively weakly on the film thickness. It should be mentioned that the results of heating-up simulations with a phase transition from the single domain state to the striped domain state at $T_{\mathrm{S}}$ below $T_{\mathrm{C}}$ agree with thermodynamical treatment of ferroelectric capacitors with dead layers by Chensky and Tarasenko. 28

For films with $d=1, T_{\mathrm{S}}$ is 150 and $210 \mathrm{~K}$ for $l=15$ and $l=31$ respectively, which is lower than the bulk transition temperature $\left(T_{\mathrm{C}} \approx 320 \mathrm{~K}\right)$. However, for $d=1$ films with $l=127$ and $l=255, T_{\mathrm{S}}$ is enhanced to 520 and $610 \mathrm{~K}$ respectively, well above the bulk $T_{\mathrm{C}}$. In the infinite thickness limit $(l \rightarrow \infty)$, it appears that $T_{\mathrm{S}}(l, d=1)$ tends to the $T_{\mathrm{C}}$ of thick films with no dead layer $(d=$ $0)$, since $T_{\mathrm{C}}$ is $650 \mathrm{~K}$ for $l=32$ and $d=0$. In the $d=1$ cases with $l=127$ and $l=255$, the effect arising from the depolarization field weakens significantly, and the enhancement of $T_{\mathrm{S}}$ results from the in-plane biaxial compressive strain. In the $d=0$ case with $l=32$, there is no depolarization field and enhancement of $T_{\mathrm{C}}$ by the inplane biaxial compressive strain is effective even in very thin films. We note that $\sqrt{\left\langle u_{z}^{2}\right\rangle}$ and $\sqrt{\left\langle u_{x}^{2}\right\rangle}$ are distinct even at high temperatures (see Fig. 4 4 ), indicating that the symmetry of the paraelectric phase is broken by the presence of the epitaxial constraint and the electrodes, as well as correlations between local dipoles and their images.

For films with a dead layer $(d=1)$, the striped domain structures appear in the cooling-down simulations at low temperatures for all values of thicknesses $l$ explored here (see Fig. [5(b) and (c) for the case of $l=15$ with $d=1$, and Fig. 6for various $l$ ). As shown in Table【, the wavevector $\boldsymbol{k}$ of the striped domain, at which $\widetilde{u}_{z}(\boldsymbol{k})$ has the largest amplitude $\left|\widetilde{u}_{z}(\boldsymbol{k})\right|$, exhibits an interesting dependence on thickness $l$. We have determined $\boldsymbol{k}$ for two 
TABLE I: Dependence of the wavevector $\boldsymbol{k} / 2 \pi$ of the striped domain structure on thickness $l$ in the thin-film $\mathrm{BaTiO}_{3}$ capacitor with a dead layer $(d=1)$.

\begin{tabular}{|c|c|c|}
\hline$l$ & $32 \times 32 \times 2(l+d)$ & $40 \times 40 \times 2(l+d)$ \\
\hline 7 & $\{4 / 323 / 320\}$ & $\left\{\begin{array}{llll}5 / 40 & 5 / 40 & 0\end{array}\right\}$ \\
\hline 15 & $\{3 / 323 / 320\}$ & $\left\{\begin{array}{llll}4 / 40 & 3 / 40 & 0\end{array}\right\}$ \\
\hline 31 & $\left\{\begin{array}{lll}2 / 32 & 2 / 32 & 0\end{array}\right\}$ & $\left\{\begin{array}{lll}3 / 40 & 2 / 40 & 0\end{array}\right\}$ \\
\hline 63 & $\left\{\begin{array}{lll}2 / 32 & 1 / 32 & 0\end{array}\right\}$ & $\left\{\begin{array}{lll}2 / 40 & 2 / 40 & 0\end{array}\right\}$ \\
\hline 127 & $\left\{\begin{array}{lll}1 / 32 & 1 / 32 & 0\end{array}\right\}$ & $\left\{\begin{array}{lll}1 / 40 & 1 / 40 & 0\end{array}\right\}$ \\
\hline 255 & $\left\{\begin{array}{lll}1 / 32 & 0 / 32 & 0\end{array}\right\}$ & $\left\{\begin{array}{lll}1 / 40 & 0 / 40 & 0\end{array}\right\}$ \\
\hline
\end{tabular}

supercell sizes, $32 \times 32 \times 2(l+d)$ and $40 \times 40 \times 2(l+d)$, to identify supercell-size effects. It can be seen that, except for the data for $l=255, \boldsymbol{k}$ tends to be along the in-plane $\{110\}$ direction, consistent with earlier reports. ${ }^{25,29}$ The simulated striped domain structure for $l=255$, which is parallel to the $\{100\}$ direction, is likely to be an artifact of the finite supercell-size: $L_{x} \times L_{y}=32 \times 32$ or even $40 \times 40$ are too small to allow for the formation of a sufficiently thick $\{110\}$ striped domain. The wavelength $\lambda=2 \pi /|\boldsymbol{k}|$ of dominant periodicity of the domain pattern is shown as a function of thickness $l$ in Fig. 7 where it is evident that the thinner films have smaller $\lambda$ to avoid the stronger depolarization field, Eq. (26). The fitting shown in Fig. 7 suggests a square-root dependence ${ }^{20}$ on $l$ (the result for $l=255$ is not included in the fit). Extensive simulations at larger length scales would probably be required to clarify further the dependence of the domain period of these striped structures on film thickness and dead-layer thickness.

The stark difference in the behavior of $\left\langle u_{z}\right\rangle$ in heatingup and cooling-down simulations hints that the (almost) uniformly polarized state and the $\left\langle u_{z}\right\rangle=0$ striped domain states are frozen and thermal hopping between them may be almost impossible at low temperatures. To understand why both uniformly polarized and striped domain states are stable and thermal hopping between them are difficult, we investigated the effective potentialenergy surfaces for striped domain structures of various stripe wavevectors $\boldsymbol{k}$ and various $l$ for thin-film ferroelectric capacitors with and without the dead layer (see Fig. 8(a)-(e)). Omitting surface relaxations in this analysis may be reasonable because the surface relaxations are confined to the surface region of the ferroelectric thin films as shown in Fig. [5(c). It can be seen that thinner ferroelectric film have a shorter stripe wavelength $\lambda=2 \pi /|\boldsymbol{k}|$ in their ground states. As the thickness $l$ is increased to $l \approx 127$, the ground state changes from the striped domain structure to the out-of-plane uniformlypolarized ferroelectric structure $(\boldsymbol{k}=(000))$. However, on the time scale of our simulations $(\approx 1 \mathrm{~ns})$, even at $l \approx 255$ there is no hopping from the striped domain metastable state to the uniformly polarized ground state (Fig. 4(d)). It can also be seen in Fig. 8(a)-(e) that the magnitude of $u_{z}$ which gives the minimum-energy ground state becomes larger, and the minimum energy gets deeper, as $l$ increases, in good correspondence with the thickness dependence of $T_{\mathrm{C}}$. The trend of $\boldsymbol{k}$ with $l$ also shows good agreement with the simulated values shown in Table [1. The simulated stability of the out-of-plane uniformly-polarized states against the energetically lower striped-domain states in thinner $(l<127)$ films at low temperature seems to give support to the recent idea of elastic stabilization of a homogeneously polarized state in strained ultrathin films ${ }^{30}$ As shown in Fig. 9)(a), the polarization switching in the epitaxially constrained film may be suppressed by the presence of a potential barrier that prevents hopping between the uniformly-polarized and striped-domain states. For $l \leq 127$ with $d=1$, it is expected that a uniformly polarized film would evolve into a striped domain state, or vice versa, over a sufficiently long time at $T<T_{\mathrm{S}}$. However, the time scale of the evolution might be very much longer than the present simulation time scale $(\sim 1 \mathrm{~ns})$. It might also be expected that, in the cooling-down simulations of films with $l \leq 127$ and $d=1$, the uniformly polarized state is obtained at $T<T_{\mathrm{S}}$. Instead, however, we find that stripes appear. A close inspection of the simulations shows that the stripes form slightly above $T_{\mathrm{S}}$, initially in a somewhat disordered fashion, presumably because such a structure provides a good compromise between energetic and entropic considerations. The stripes then get frozen into place, and become better ordered, as the temperature is reduced below $T<T_{\mathrm{S}}$. Conversely, in the case of $d=0$ (Fig. 4(e), depolarization field $\mathcal{E}_{\mathrm{d}}=0$ ), the striped domain stricture does not appear during the heating-up and cooling-down simulations. This may be because, when $\mathcal{E}_{\mathrm{d}}=0$, there is no reason or chance to form a striped domain structure even just above $T_{\mathrm{C}}$. At $T_{\mathrm{C}}$, direct phase transition form paraelectric phase to uniformly polarized ferroelectric phase occurs. Then, below $T_{\mathrm{C}}$, the system tends to be in its ground state, the uniformly polarized ferroelectric structure.

\section{Hysteresis loops}

A measurement of polarization typically involves use of a triangle-wave electric field for recording the ferroelectric hysteresis loops (inset of Fig. 10). The hysteresis loops and coercive fields $\mathcal{E}_{\mathrm{c}}$ depend on the amplitude $\mathcal{E}_{0}$ and frequency $f$ of the applied fields. We simulate hysteresis here using triangle-wave with steps (width $\Delta t n_{\text {steps }}$ and height $\Delta \mathcal{E}$ ) as sketched schematically in Fig. 10. Thus, the frequency of the applied field in our simulations is $f=\Delta \mathcal{E} / 4 \Delta t n_{\text {steps }} \mathcal{E}_{0}$. We used supercell sizes of $L_{x} \times L_{y} \times L_{z}=16 \times 16 \times 2(l+d)$ in simulations of hysteresis loops for ferroelectric thin-film capacitors with $1 \%$ in-plane biaxial compressive strain and without constraints of strain (namely, the "free" film) (see Fig. 11). The temperature is maintained at $100 \mathrm{~K}$ through the simulations. For both the epitaxially constrained and "free" films, our simulations confirm that the imperfect screening of the electrodes decreases the coercive field as 
the film thicknesses decreases, as described phenomenologically in Ref. [8]. There is a large (order-of-magnitude) difference in the coercive field $\mathcal{E}_{\mathrm{c}}$ between the epitaxially constrained film and the "free" film. This may be because the compressive strain arising from epitaxial constraints prevents the polarization switching, while the inclusion of inhomogeneous strain (i.e., acoustic displacements) eases the switching, as depicted in Fig. 9. The potential barriers themselves are lower in the "free" films than in the epitaxially constrained films (see Fig. 8). We note that hysteresis loops for "free" film capacitors with $l=63$ and $l=127$ are very similar to the experimentally observed hysteresis loops of a ferroelectric capacitor with damaged electrodes that have "steps" and "plateaus" during polarization switchings.$\underline{1}$ This is because, in the "free" film capacitors with imperfect electrodes $(d=1)$, the configuration with out-of-plane polarization is no longer the ground state. In fact, the ground state has a nonzero in-plane polarization. Thus, the dipoles $Z^{*} \boldsymbol{u}(\boldsymbol{R})$ have large in-plane components $Z^{*} u_{x}(\boldsymbol{R})$ and $Z^{*} u_{y}(\boldsymbol{R})$ in the hysteresis-loop simulations (and experiments), as evident in the snapshot shown in Fig. 12 .

Unfortunately, attempts to fit our results to the usually-assumed Kay-Dunn scaling of the coercive field $\mathcal{E}_{\mathrm{c}}$ with film thickness $l$ of thicker films ${ }^{31}$ were unsuccessful, as were attempts to emulate the relatively weak dependence of $\mathcal{E}_{\mathrm{c}}$ on $l$ for epitaxially grown high-quality ultrathin films $32,33,34$ The experimentally observed values of coercive fields $\mathcal{E}_{\mathrm{c}}$ for ultrathin $\mathrm{BaTiO}_{3}$ capacitors range from 200 to $500 \mathrm{kV} / \mathrm{cm}, 32,33,34$ while simulations of epitaxially constrained films largely overestimate $\mathcal{E}_{\mathrm{c}}$, and those of "free" films slightly underestimate $\mathcal{E}_{\mathrm{c}}$. This may be because the switching in real thin-film capacitors is a large-scale $(>100 \mathrm{~nm})$ phenomenon involving defect-mediated nucleation mainly at ferroelectrics-electrodes interfaces,$\stackrel{18,31,35,36,37,38}{ }$ as well as the possibility that the strain conditions may be intermediate between the cases of epitaxially constrained and "free" films. Such intermediate strain conditions may be achieved and will be simulated with MD in the future by introducing a mechanical boundary condition such as presented in Ref. [30]. In contrast to our case of ultrathin $\mathrm{BaTiO}_{3}$ capacitors, it is well known that for ultrathin $\overline{\mathrm{PbZr}}_{x} \mathrm{~T}_{1-x} \mathrm{O}_{3}$ (PZT) capacitors the coercive fields $\mathcal{E}_{\mathrm{c}}$ $\underline{\text { increase with decreasing film thickness } l \text {, and there is an }}$ argument whether this strong increase of $\mathcal{E}_{\mathrm{c}}$ is coming from compressive substrate-induced lattice strain $^{39}$ or not $\underline{40}$ Constructing a first-principles Hamiltonian for PZT and simulations with this MD method will help us to understand this difference between $\mathrm{BaTiO}_{3}$ and PZT.

\section{SUMMARY}

We have developed a robust and highly efficient molecular-dynamics scheme, based on a first-principles effective Hamiltonian formulation, for simulating the behavior of the polarization in perovskite-type ferroelectrics. We have applied this approach to study $\mathrm{BaTiO}_{3}$ ferroelectric thin-film capacitors, with special attention to the dependence on film thickness and choice of electric boundary conditions. We find that striped domain structures tend to form on cooling-down simulations when a ferroelectric dead layer is present near the electrodes, and we study the dependence of the domain period on the conditions of formation. We also study the hysteresis loops for capacitor structures, both with and without such dead layers, and we find dramatic differences in the hysteretic behavior for the cases of elastically constrained or "free" films. Our MD simulator feram will be a powerful tool for further investigations of the physical properties of ferroelectric nanostructures that are relevant for a variety of potential device applications.

\section{Acknowledgments}

Computational resources were provided by the Center for Computational Materials Science, Institute for Materials Research (CCMS-IMR), Tohoku University. We thank the staff at CCMS-IMR for their constant effort. This research was done when T.N. stayed at JNCASR and Rutgers University under the support from JNCASR, Rutgers University, the Ministry of Education, Culture, Sports, Science and Technology (MEXT) of Japan, and the Japan Society for the Promotion of Science (JSPS). D.V. acknowledges support of ONR Grant N00014-05-1-0054.
1 J. F. Scott, Ferroelectric Memories (Springer-Verlag, Berlin Heidelberg, 2000).

2 K. J. Choi, M. Biegalski, Y. L. Li, A. Sharan, J. Schubert, R. Uecker, P. Reiche, Y. B. Chen, X. Q. Pan, V. Gopalan, et al., Science 306, 1005 (2004).

3 M. E. Drougard and R. Landauer, J. Appl. Phys. 30, 1663 (1959).

4 S. L. Miller, R. D. Nasby, J. R. Schwank, M. S. Rodgers, and P. V. Deessendorger, J. Appl. Phys. 68, 6463 (1990).
5 V. V. Lemanov and V. K. Yarmarkin, Phys. Solid State 38, 1363 (1996), [Fiz. Tverd. Tela 38, 2482 (1996)].

${ }^{6}$ H. Z. Jin and J. Zhu, J. Appl. Phys. 92, 4594 (2002).

7 R. R. Mehta, B. D. Silverman, and J. T. Jacobs, J. Appl. Phys. 44, 3379 (1973).

8 M. Dawber, P. Chandra, P. B. Littlewood, and J. F. Scott, J. Phys.-Condes. Matter 15, L393 (2003).

9 R. D. King-Smith and D. Vanderbilt, Phys. Rev. B 49, 5828 (1994). 
10 W. Zhong, D. Vanderbilt, and K. M. Rabe, Phys. Rev. Lett. 73, 1861 (1994).

11 W. Zhong, D. Vanderbilt, and K. M. Rabe, Phys. Rev. B 52, 6301 (1995)

12 U. V. Waghmare and K. M. Rabe, Phys. Rev. B 55, 6161 (1997).

13 U. V. Waghmare, E. J. Cockayne, and B. P. Burton, Ferroelectrics 291, 187 (2003).

14 B. P. Burton, E. Cockayne, and U. V. Waghmare, Phys. Rev. B 72, 064113 (2005).

15 J. Paul, T. Nishimatsu, Y. Kawazoe, and U. V. Waghmare, Phys. Rev. Lett. 99, 077601 (2007).

16 S. D. Bond, B. J. Leimkuhler, and B. B. Laird, J. Comput. Phys. 151, 114 (1999).

17 N. Sai, A. M. Kolpak, and A. M. Rappe, Phys. Rev. B 72, 020101 (2005).

18 D. J. Kim, J. Y. Jo, Y. S. Kim, Y. J. Chang, J. S. Lee, J.-G. Yoon, T. K. Song, and T. W. Noh, Phys. Rev. Lett. 95, 237602 (2005).

19 G. Gerra, A. K. Tagantsev, N. Setter, and K. Parlinski, Phys. Rev. Lett. 96, 107603 (2006).

20 C. Kittel, Phys. Rev. 70, 965 (1946).

21 D. D. Fong, G. B. Stephenson, S. K. Streiffer, J. A. Eastman, O. Auciello, P. H. Fuoss, and C. Thompson, Science 304, 1650 (2004).

22 A. M. Bratkovsky and A. P. Levanyuk, Phys. Rev. Lett. 84, 3177 (2000).

23 A. M. Bratkovsky and A. P. Levanyuk, Phys. Rev. Lett. 87, 179703 (2001).

24 B. K. Lai, I. Ponomareva, I. I. Naumov, I. Kornev, H. X. Fu, L. Bellaiche, and G. J. Salamo, Phys. Rev. Lett. 96, 137602 (2006).

25 B.-K. Lai, I. Ponomareva, I. A. Kornev, L. Bellaiche, and G. J. Salamo, Phys. Rev. B 75, 085412 (2007).

26 B.-K. Lai, I. Ponomareva, I. Kornev, L. Bellaiche, and G. Salamo, Appl. Phys. Lett. 91, 152909 (2007).

27 See EPAPS Document No. XXXXX for animations of the cooling-down and heating-up simulations. This document can be reached through a direct link in the online article's HTML reference section or via the EPAPS homepage http://www.aip.org/pubservs/epaps.html Before PRB accept this manuscript, animations are temporally placed at http://www-lab.imr.tohoku.ac.jp/\%7Et-nissie/animations/ .

28 E. V. Chensky and V. V. Tarasenko, Sov. Phys. JETP 56, 618 (1982).

29 S. Tinte and M. G. Stachiotti, Phys. Rev. B 64, 235403 (2001).

30 N. A. Pertsev and H. Kohlstedt, Phys. Rev. Lett. 98, 257603 (2007)

31 H. F. Kay and J. W. Dunn, Phil. Mag. 7, 2027 (1962).

${ }^{32}$ Y. S. Kim, J. Y. Jo, D. J. Kim, Y. J. Chang, J. H. Lee, T. W. Noh, T. K. Song, J.-G. Yoon, J. S. Chung, S. I. Baik, et al., Appl. Phys. Lett. 88, 072909 (2006).

33 J. Y. Jo, Y. S. Kim, T. W. Noh, J.-G. Yoon, and T. K. Song, Appl. Phys. Lett. 89, 232909 (2006).

34 A. Petraru, N. A. Pertsev, H. Kohlstedt, U. Poppe, R. Waser, A. Solbach, and U. Klemradt, J. Appl. Phys. 101, 114106 (2007).

35 V. Janovec, Czech. J. Phys. 8, 3 (1958).

36 P. Chandra, M. Dawber, P. B. Littlewood, and J. F. Scott, Ferroelectrics 313, 7 (2004).

37 A. K. Tagantsev and G. Gerra, J. Appl. Phys. 100, 051607 (2006).

38 J. Y. Jo, D. J. Kim, Y. S. Kim, S.-B. Choe, T. K. Song, J.-G. Yoon, and T. W. Noh, Phys. Rev. Lett. 97, 247602 (2006).

39 N. A. Pertsev, J. R. Contreras, V. G. Kukhar, B. Hermanns, H. Kohlstedt, and R. Waser, Appl. Phys. Lett. 83, 3356 (2003).

${ }^{40}$ H. N. Lee, S. M. Nakhmanson, M. F. Chisholm, H. M. Christen, K. M. Rabe, and D. Vanderbilt, Phys. Rev. Lett. 98, 217602 (2007). 


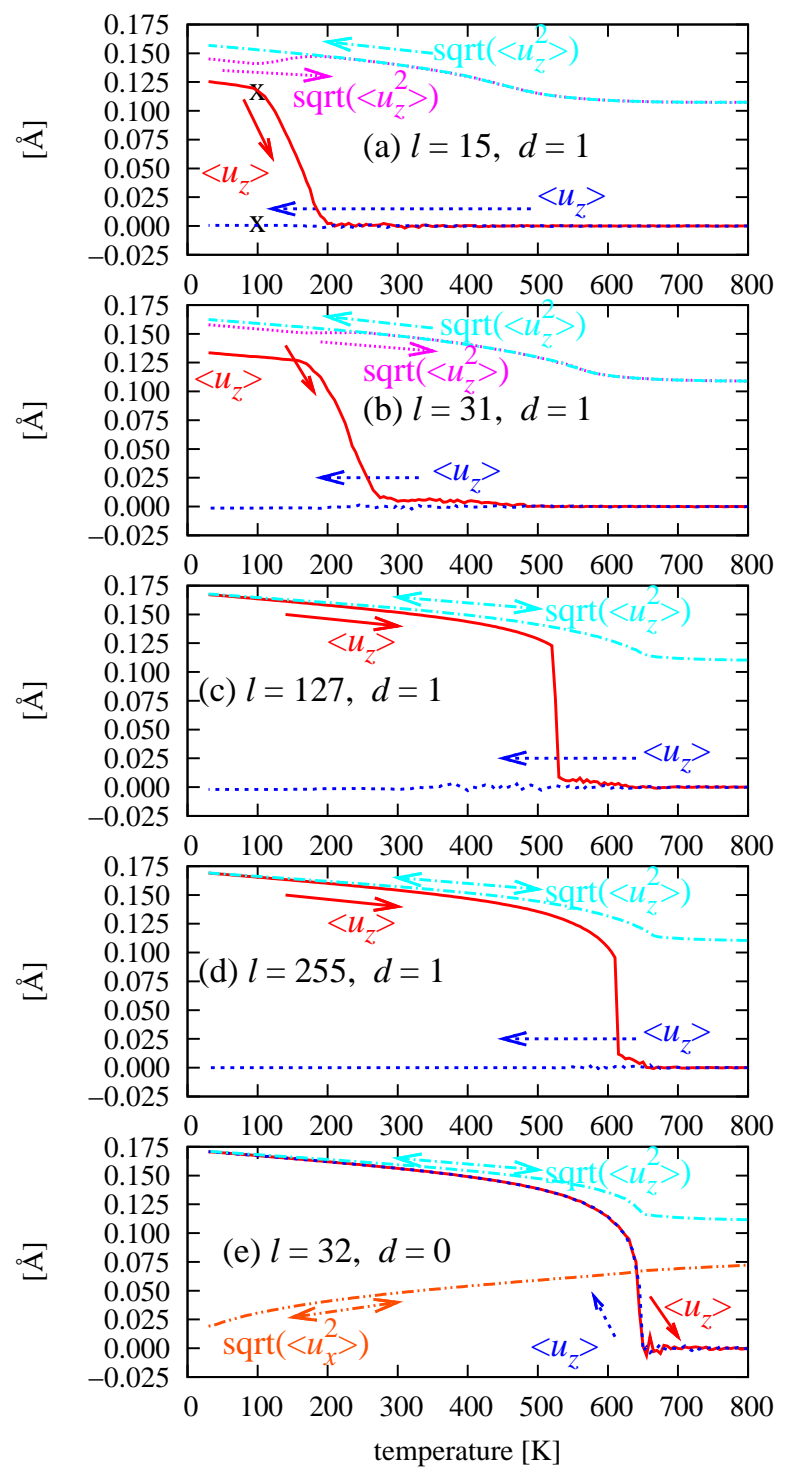

FIG. 4: $\left\langle u_{z}\right\rangle$ of heating-up (solid lines) and cooling-down (dashed lines) molecular-dynamics simulations of $\mathrm{BaTiO}_{3}$ thin-film capacitors with short-circuited electrodes under $1 \%$ in-plane biaxial compressive strain for (a) thickness $l=$ 15 layer with dead layer $d=1$, (b) $l=31$ with $d=1$, (c) $l=127$ with $d=1$, (d) $l=255$ with $d=1$, and (e) $l=32$ without dead layer $(d=0) . \sqrt{\left\langle u_{z}^{2}\right\rangle}$ are also plotted in (a)(e). In (c)-(e), heating-up $\sqrt{\left\langle u_{z}^{2}\right\rangle}$ and cooling-down $\sqrt{\left\langle u_{z}^{2}\right\rangle}$ are almost identical. $\sqrt{\left\langle u_{x}^{2}\right\rangle}$ is plotted only in (e), because the behaviors of $\sqrt{\left\langle u_{x}^{2}\right\rangle}$ and $\sqrt{\left\langle u_{y}^{2}\right\rangle}$ are essentially identical in cases (a)-(e) (for both heating-up and cooling-down). Supercells are of size $32 \times 32 \times 2(l+d)$. Animations of these cooling-down and heating-up simulations are also available in the EPAPS. ${ }^{27}$ 

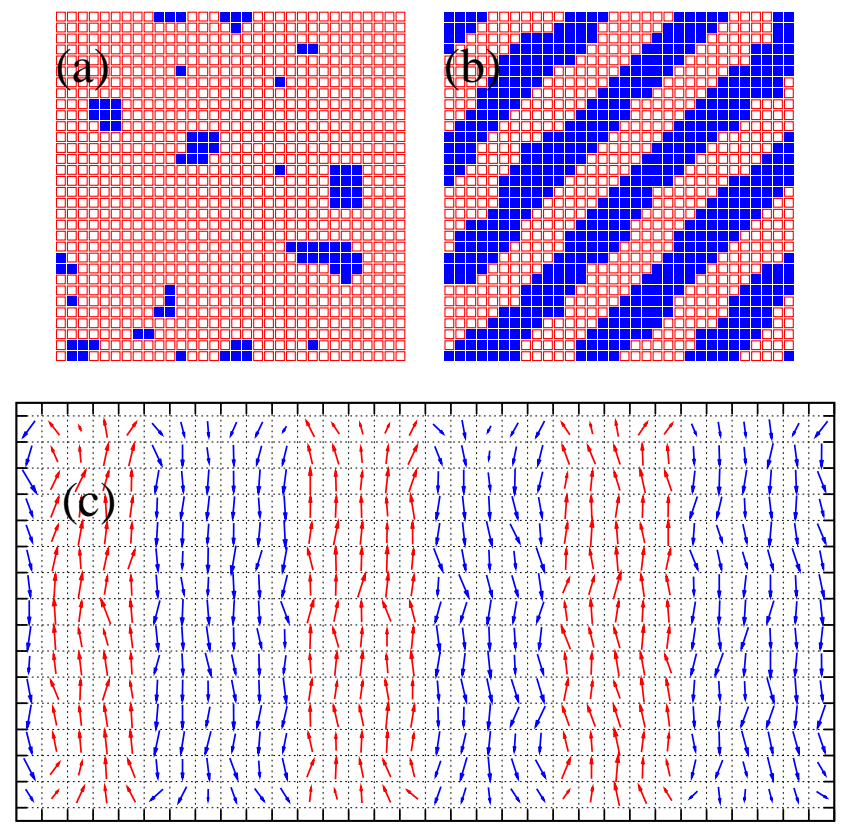

FIG. 5: Snapshots at $T=100 \mathrm{~K}$ in heating-up ((a)) and cooling-down ((b) and (c)) simulations of ferroelectric thinfilm capacitors of $l=15$ with $d=1$. (a) and (b) are horizontal slices. (c) is a vertical cross section. Points of snapshots are indicated with "X" marks in Fig. 4(a). In horizontal slices, the $+z$-polarized and $-z$-polarized sites are denoted by $\square$ and $\boldsymbol{\square}$, respectively. In vertical cross sections, the dipole moments of each site are projected onto the $x z$-plane and indicated with arrows. Layers which do not have arrows are dead layers.

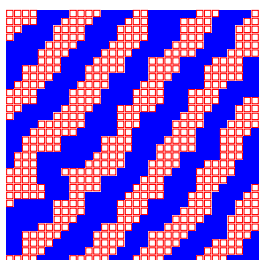

(a) $l=7$

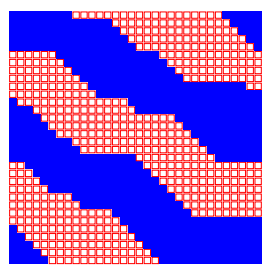

(d) $l=63$

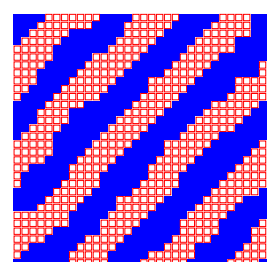

(b) $l=15$

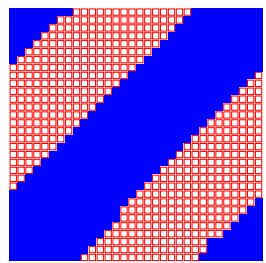

(e) $l=127$

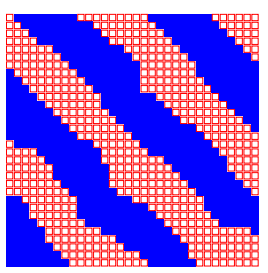

(c) $l=31$

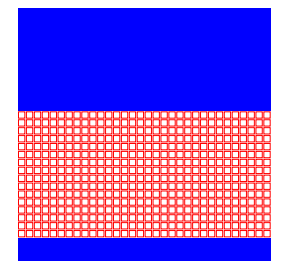

(f) $l=255$

FIG. 6: Horizontal slices of snapshots at $100 \mathrm{~K}$ in coolingdown simulations of ferroelectric thin-film capacitors with single dead layer $(d=1)$ of various thickness $l=7,15,31,63$, 127 , and 255 . The $+z$-polarized and $-z$-polarized sites are denoted by $\square$ and $\boldsymbol{\square}$, respectively. 


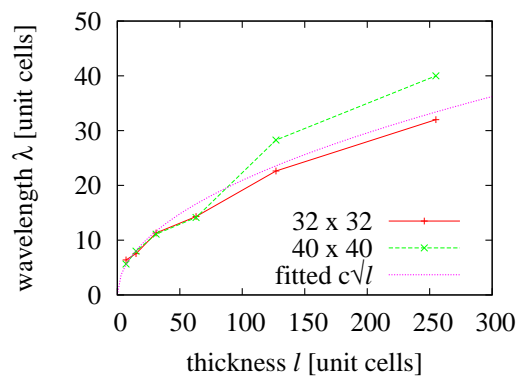

FIG. 7: Calculated thickness $l$ dependence of wavelength $\lambda$ of striped domain structures in thin film $\mathrm{BaTiO}_{3}$ capacitors with a dead layer $(d=1) .+$ marks are from $32 \times 32 \times 2(l+d)$ supercell calculations and $\times$ are those of $40 \times 40 \times 2(l+d)$. Data of $l<=127$ are fitted with $\lambda=c \sqrt{l}$ (dotted line). $l=255$ data are omitted, because of their large supercell-size dependence. 


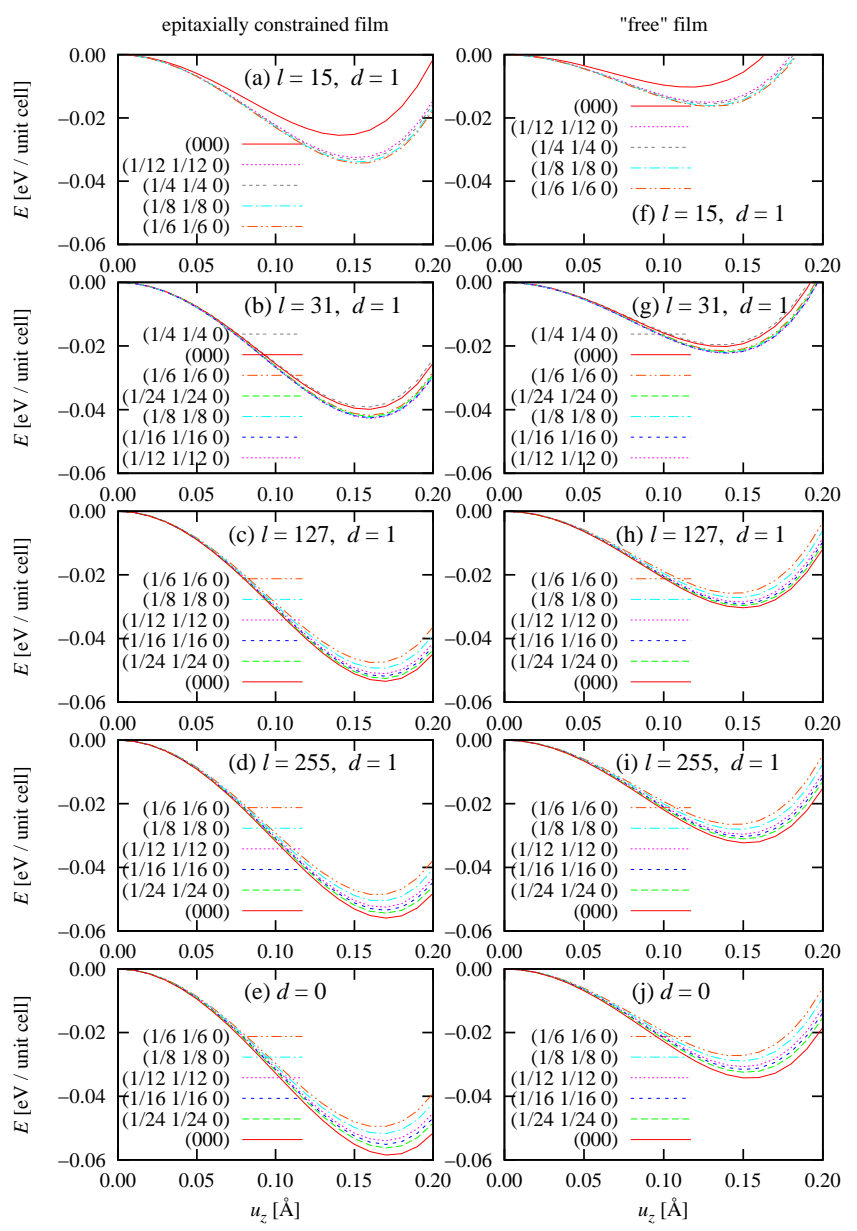

FIG. 8: Effective potential surfaces of $\mathrm{BaTiO}_{3}$ thin-film capacitors with short-circuited electrodes: (a)-(e), under $1 \%$ in-plane biaxial compressive strain arising from epitaxial constraints; (f)-(j), without epitaxial constraints (i.e., for "free" films). The thicknesses of ferroelectric films and dead layers are indicated in each panel with $l$ and $d$ respectively. Total energies as functions of $u_{z}$ are compared among striped domain structures with wavevectors $\boldsymbol{k}$ parallel to (110). $\boldsymbol{k}=(000)$ corresponds to the uniformly polarized structure. The zero of the energy scale is placed at the total energy of the nonpolarized $u_{z}=0$ structure. A negative pressure $p=-5 \mathrm{GPa}$ is applied to correct the underestimation in $T_{\mathrm{C}}$. 

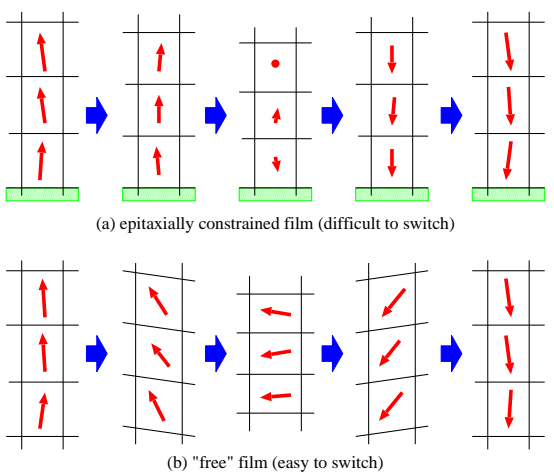

FIG. 9: Schematic comparison between the epitaxially constrained film and the "free" film. In the epitaxially constrained film, switching may have to climb over a potential barrier, but, in the "free" film, dipoles can be easily rotated and switching can go around a valley of the potential.

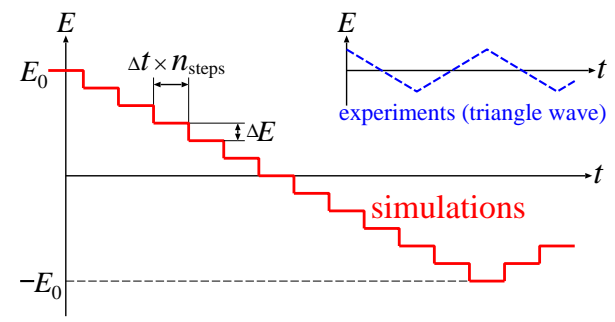

FIG. 10: Schematic illustrations of triangle-wave electric field used to measure ferroelectric hysteresis loops experimentally (inset) and in the present simulations.

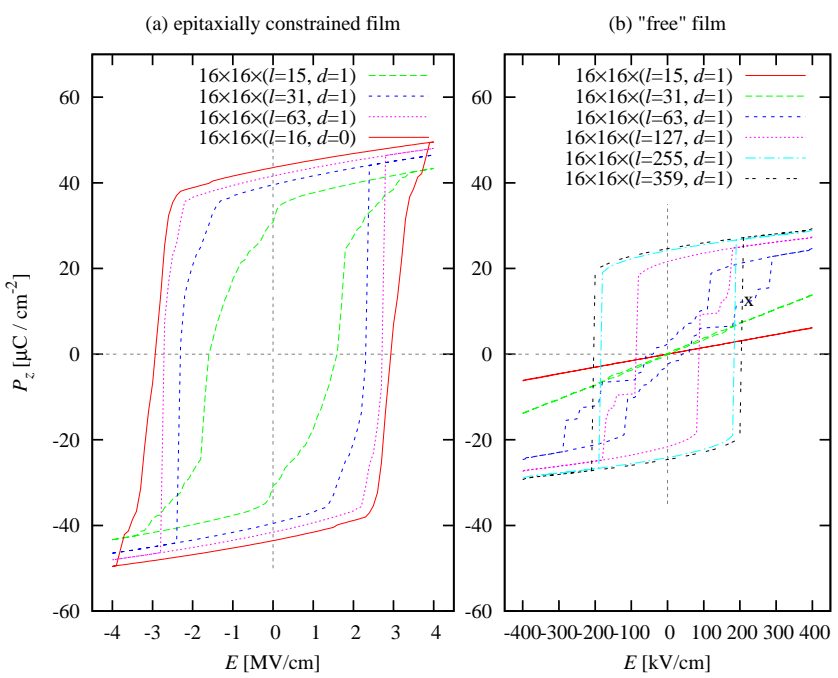

FIG. 11: Calculated hysteresis loops for capacitors with (a) epitaxially constrained films, and (b) "free" films of various thickness $l$ and with dead layer $d$. Supercell sizes were $16 \times 16 \times 2(l+d) . \Delta t=2$ fs and $n_{\text {steps }}=50,000$. For epitaxially constrained films, $\mathcal{E}_{0}=4,000 \mathrm{kV} / \mathrm{cm}$ and $\Delta \mathcal{E}=100 \mathrm{kV} / \mathrm{cm}$ are employed. For "free" films, $\mathcal{E}_{0}=400 \mathrm{kV} / \mathrm{cm}$ and $\Delta \mathcal{E}=10 \mathrm{kV} / \mathrm{cm}$ are employed. 


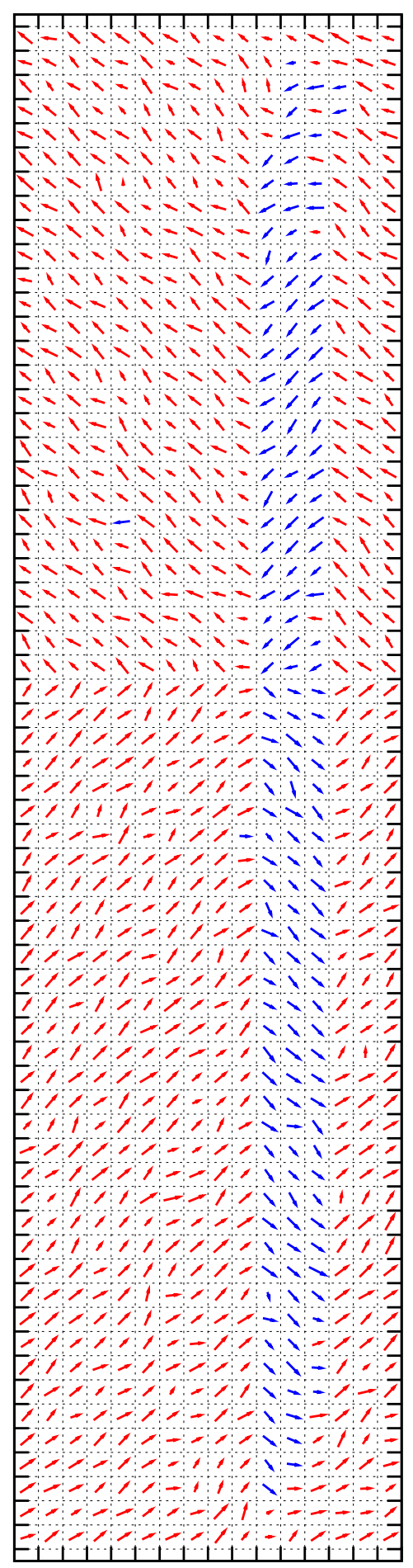

FIG. 12: Vertical cross section of a simulated ferroelectric "free" film capacitor with a single dead layer; $16 \times 16 \times(l=$ $63, d=1)$. The snapshot was taken at the point marked " $\mathrm{x}$ " in Fig. 11(b). The projection of the dipole moments onto the $x z$-plane are indicated with arrows. 